\title{
Studying Mass Transfer in Glasses using Cathodoluminescence.
}

\author{
Peter McSwiggen ${ }^{1}$ and Colin M. MacRae ${ }^{2}$ \\ ${ }^{1}$ McSwiggen \& Associates / JEOL USA, Inc., St. Anthony, MN, U.S.A. \\ ${ }^{2}$ Microbeam Laboratory, CSIRO Minerals, Clayton, Victoria 3168, Australia
}

Optical fibers are formed through a complex manufacturing process. Many types of optical fibers are manufactured. The details depend on their designed task. Some are designed for simple telecommunication functions, others act as amplifiers, and some are designed to maintain the polarization of the light as it passes along its length. There are some common features, though. Optical fibers are designed such that light photons traveling down the core of the fiber will reflection off the interface between the core and cladding, keeping the light contained within the core. The core and cladding are glasses of two different compositions, with the core having the higher refractive index. This difference in refractive index causes the total reflection at the interface. Surrounding the working portion of the fiber, the remainder of the fiber is typically pure $\mathrm{SiO}_{2}$ glass. This material started out as a hollow glass tube, during the initial stage of manufacturing. Using a chemical vapor deposition process, the working components of the desired fiber are deposited sequentially onto the inner surface of the hollow tube. This is done by passing the desired chemicals, in a vapor form, though the tube. While the tube is slowly rotated horizontally on a lathe, the external surface is heated to $1600^{\circ} \mathrm{C}\left(3000^{\circ} \mathrm{F}\right)$. The chemicals react with the oxygen in the tube, and are deposited as sooty, oxide particles. Once the required layers have been deposited, the hollow tube is collapsed into a solid glass rod, called the preform. This process is accomplished by concentrating the heat, for a longer period, on the external surface of the tube, while it continues to rotate. The tube slowly redistributed its mass, while its diameter decreases and the thickness of the walls increase. Eventually it becomes one solid glass rod.

Intuitively one might think that this process of redistributing the mass within the walls of the glass tube would be a chaotic, disorganized process. However, observations show that the exact opposite is true. Cathodoluminescence (CL) imaging of the outer glass shows that it has separated into layers, and each layer has segregated into elliptical, concentric zones (Fig. 1). The number of concentric zones is always six per layer. The dimensions of these concentric zones vary dramatically, but there are always six elliptical rings per layer. This zoning can only be seen in the CL signal. Conventional, $\mathrm{X}$-ray elemental mapping shows no variation in the composition of the glass, since it was made from high purity $\mathrm{SiO}_{2}$. However, the variation in the CL intensity might be the result of trace compositional differences that are in concentrations below the detection limits of electron probe microanalyzers (EPMA). Full spectrum mapping of the preforms show that each of the different components of the glass rod has a very different CL spectrum (Fig. 2).

The concentric, elliptical zones must represent the method by which the mass of the glass is transferred from the outer regions of the glass tubing inwards as the diameter collapses. It is not known whether outward layering previously existed in the original glass tubes, or whether they also developed during the collapse of the tube. In either case, CL imaging gives dramatic insights into the mechanism by which the mass of the tubing redistributed itself while it is compressed into a glass rod. Samplings and CL imaging at various stages of this transition could explain in better detail this mass transfer mechanism. 

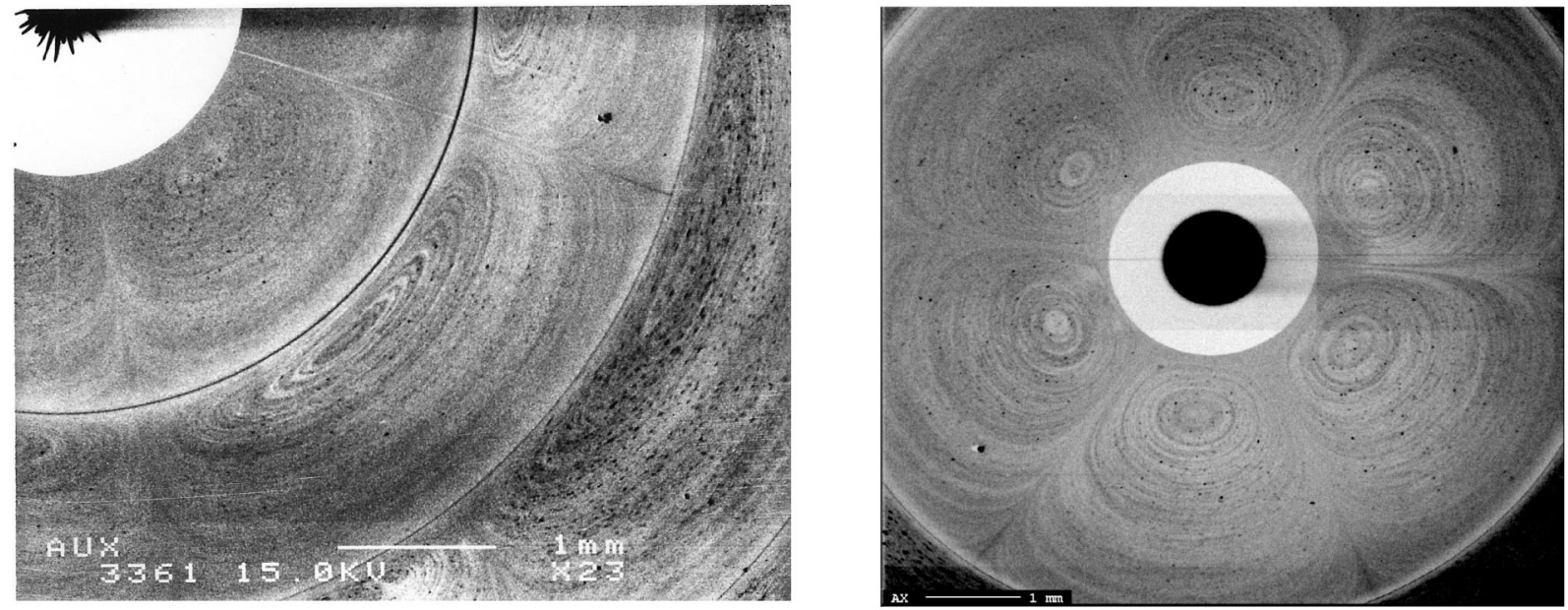

Figure 1. Examples of concentric zoning in optical fiber preforms as seen in their total intensity cathodoluminescence (CL) signal. Variations in the intensity illustrates the zoning that occurs as a result of mass transfer during the collapse of the original tube into a glass rod. The CL signal is inverted. Dark areas have the highest signal intensity.

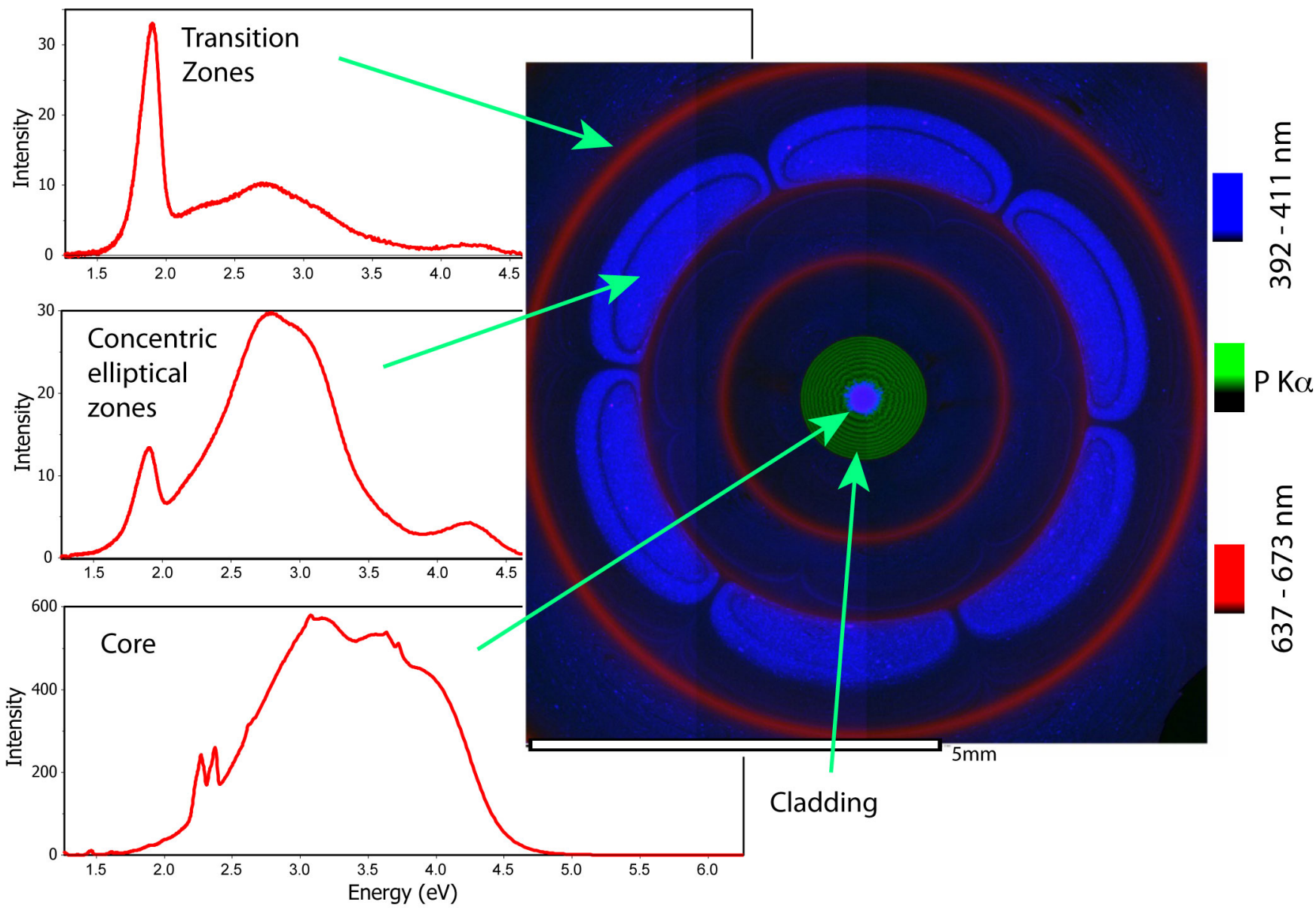

Figure 2. Full spectrum CL mapping of a optical fiber preform. Map combines the intensity of two segments of the CL spectrum with the intensity of phosphorous (right). To the left are representative spectra from the various zones within the preform. 